Trauma Berufskrankh 2010 - 12 [Suppl 1]:29-37 DOI 10.1007/s10039-009-1508-z

Online publiziert: 19. August 2009

(c) Springer Medizin Verlag 2009

B. Herbst · H.-R. Kortmann

BG-Unfallklinik Duisburg GmbH

\title{
Postoperative ambulante Verordnungen
}

\section{Krankengymnastik/ physikalische Therapien}

Gesetzlichen Unfallversicherung (DGUV) [1] ist festgelegt, wer im berufsgenossenschaftlich ambulanten Heilverfahren die ambulanten Verordnungen ausstellt: die niedergelassenen Durchgangs- (D-) und $\mathrm{H}$-Ärzte (H: an der Heilbehandlung beteiligt), die zum Verletztenartenverfahren (VAV) zugelassenen VAV-Krankenhäuser und die BG-Unfallkliniken.

Verordnet werden vornehmlich Physiotherapien (PT), Krankengymnastik (KG), Erweiterte Ambulante Physiotherapie (EAP) mit Medizinischer Trainingstherapie (MTT) sowie berufsrehabilitative Trainingseinheiten als Ergotherapie (ET), berufsorientierte (BOR) oder arbeitsplatzspezifische Rehabilitationen (ASR), darüber hinaus alle erforderlichen postoperativen Hilfsmittel für die ambulante Versorgung, wie orthopädische Schuhe, Orthesen, Prothesen oder Rollstühle.

Mit den Verordnungen über Physiotherapien, Krankengymnastik und physikalische Therapien sind die Behandlungsziele vorgegeben:

- Verbesserung der Beweglichkeit verletzter und/oder mitbetroffener $\mathrm{Ge}$ lenke,

- Aufbau reduzierter Muskelkraft,

- Lösen von Weichteilverwachsungen und Narbengewebe,

- Dehnen verkürzter Muskulatur,

- Lockern verspannter Muskulatur,

- Schmerzlinderung durch Massagen, Wärme- und Kältetherapie,

- Gang- und Gebrauchsschulungen,

- Stabilisationstraining,

- Verbesserung des Koordinationsund Gleichgewichtsvermögens,

- Belastungs- und Leistungstraining.
Mit dem Formblatt F2400 der DGUV sind die Verordnungen von Leistungen zur Krankengymnastik und physikalischen Therapie standardgemäß festgelegt. Die Leistungsziffern und -beschreibungen sind in 5 Sparten unterteilt, die sich in der Art ihrer Behandlungsanwendungen unterscheiden:

- die 81er (91er) Ziffern für die Verordnungen von krankengymnastischen Behandlungen inklusive Krankengymnastik im Bewegungsbad und manuellen Therapien,

- die 82er (92er) Ziffern für die Verordnungen physikalischer Therapien (Wärme- und Kälteanwendungen),

- die 83er (93er) Ziffern für die Verordnungen von Elektrotherapien, Ultraschall und Iontophorese,

- die 84er (94er) Ziffern für die Verordnungen von Massagen, Lymphdränagen, Kompressionsbehandlungen,
Teilbädern, Chirogymnastik und Extensionbehandlungen,

- die 85er (95er) Ziffern für die Verordnungen von Inhalationen, Ruheintervall und ärztlich verordneten Hausbesuchen.

Die Behandlungszeitintervalle variieren zwischen 1 und 4 Zeiteinheiten á $10 \mathrm{~min}$. Im Regelfall wird eine Verordnung für die festgelegte Behandlungszeit von 2-4 Wochen á 2-3 Behandlungseinheiten wöchentlich ausgestellt. Ärztliche Kontrolluntersuchungen sind im Zeitabstand von längstens 14 Tagen vorgesehen. Weiterverordnungen nach einer Behandlungszeit von 4 Wochen bedürfen der ärztlichen Begründung gegenüber dem Unfallversicherungsträger.

\section{CPM ("continous passive motion")}

Durch die Verlagerung langer Hospitalisierungszeiten zugunsten frühpostoperativer, ambulanter Weiterbehandlungen

\begin{tabular}{|c|c|c|c|c|}
\hline Operation & Hüft-TEP & Knie-TEP & Schulter-TEP & Ellenbogen-TEP \\
\hline CPM-Schiene & $\begin{array}{l}\text { Ab 1. postope- } \\
\text { rativem Tag, } \\
\text { solange Hüft- } \\
\text { beugung }<90^{\circ}\end{array}$ & $\begin{array}{l}\text { Ab 1. postopera- } \\
\text { tivem Tag, solan- } \\
\text { ge Kniebeugung } \\
<90^{\circ}\end{array}$ & $\begin{array}{l}\text { Ab 1. postopera- } \\
\text { tivem Tag, solan- } \\
\text { ge Schultereleva- } \\
\text { tion }<90^{\circ}\end{array}$ & $\begin{array}{l}\text { Ab 1. postopera- } \\
\text { tivem Tag, solange } \\
\text { Ellenbogen- } \\
\text { beugung }<90^{\circ}\end{array}$ \\
\hline Motomed & $\begin{array}{l}\text { Ab 2. postope- } \\
\text { rativer Woche, } \\
\text { wenn Hüft- } \\
\text { beugung }>70^{\circ}\end{array}$ & $\begin{array}{l}\text { Ab 3. postope- } \\
\text { rativer Woche, } \\
\text { wenn Kniebeu- } \\
\text { gung }>70^{\circ}\end{array}$ & & \\
\hline $\begin{array}{l}\text { Ergometer (Hand-/ } \\
\text { Fußpedale) }\end{array}$ & $\begin{array}{l}\text { Ab 3. postope- } \\
\text { rativer Woche, } \\
\text { wenn Hüft- } \\
\text { beugung }>90^{\circ}\end{array}$ & $\begin{array}{l}\text { Ab 4. postope- } \\
\text { rativer Woche, } \\
\text { wenn Knie- } \\
\text { beugung }>90^{\circ}\end{array}$ & $\begin{array}{l}\text { Ab 3. postope- } \\
\text { rativer Woche, } \\
\text { wenn Schultere- } \\
\text { levation }>90^{\circ}\end{array}$ & $\begin{array}{l}\text { Ab 4. postopera- } \\
\text { tiver Woche, wenn } \\
\text { Ellenbogen- } \\
\text { beugung }>90^{\circ}\end{array}$ \\
\hline
\end{tabular}




\section{Postoperative ambulate Verordnungen}
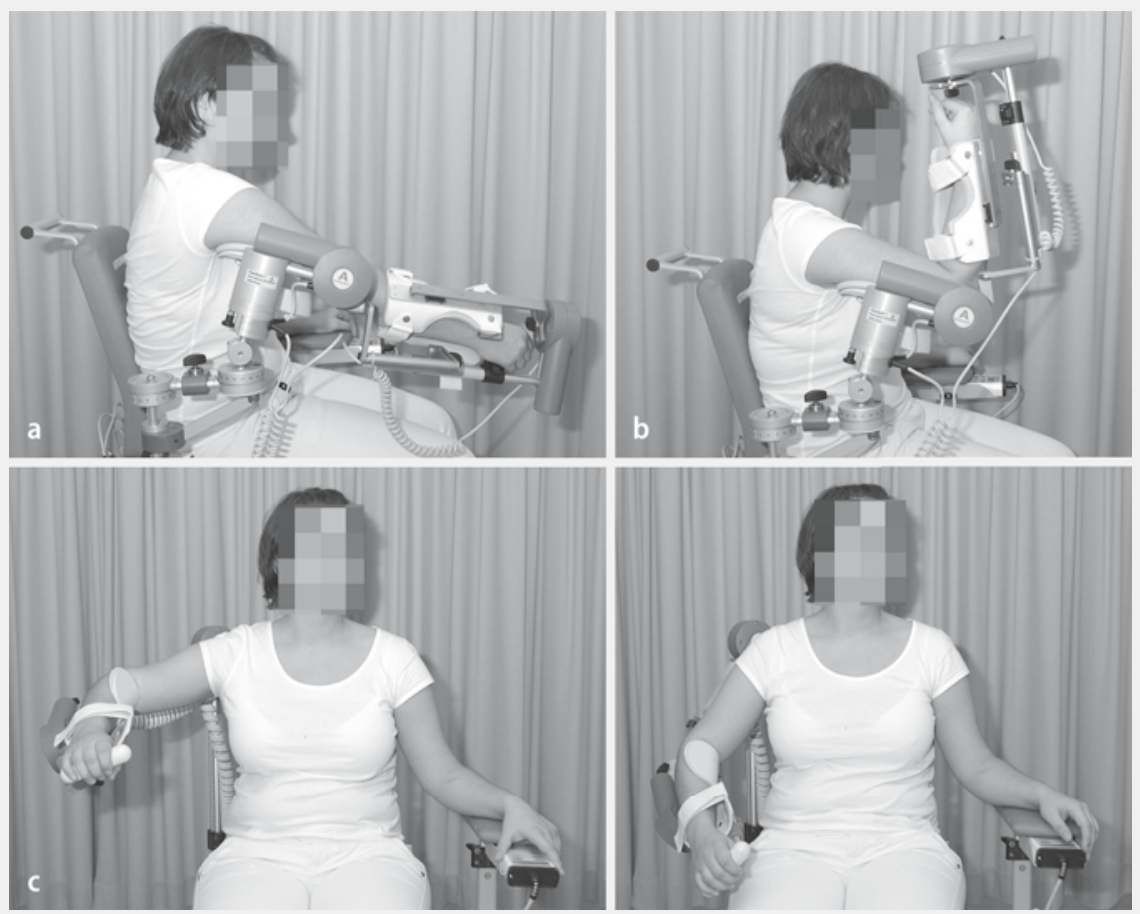

Abb. 14 CPM-Arm-Motorschiene, a Extension, b Flexion, c Abduktion, d Adduktion

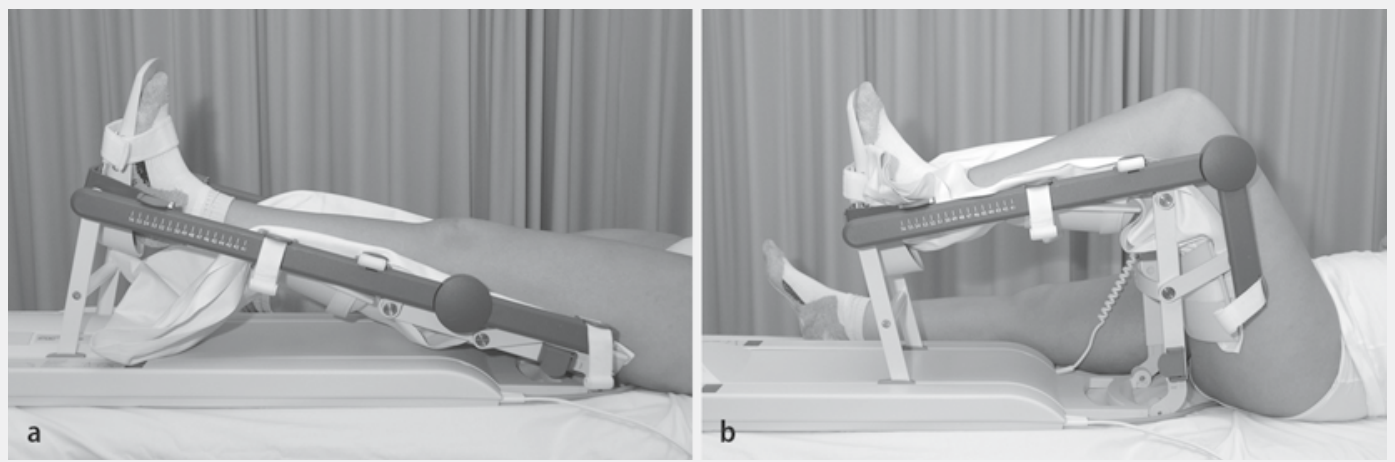

Abb. $2<$ CPM-Bein-

Motorschiene, a Extension, b Flexion
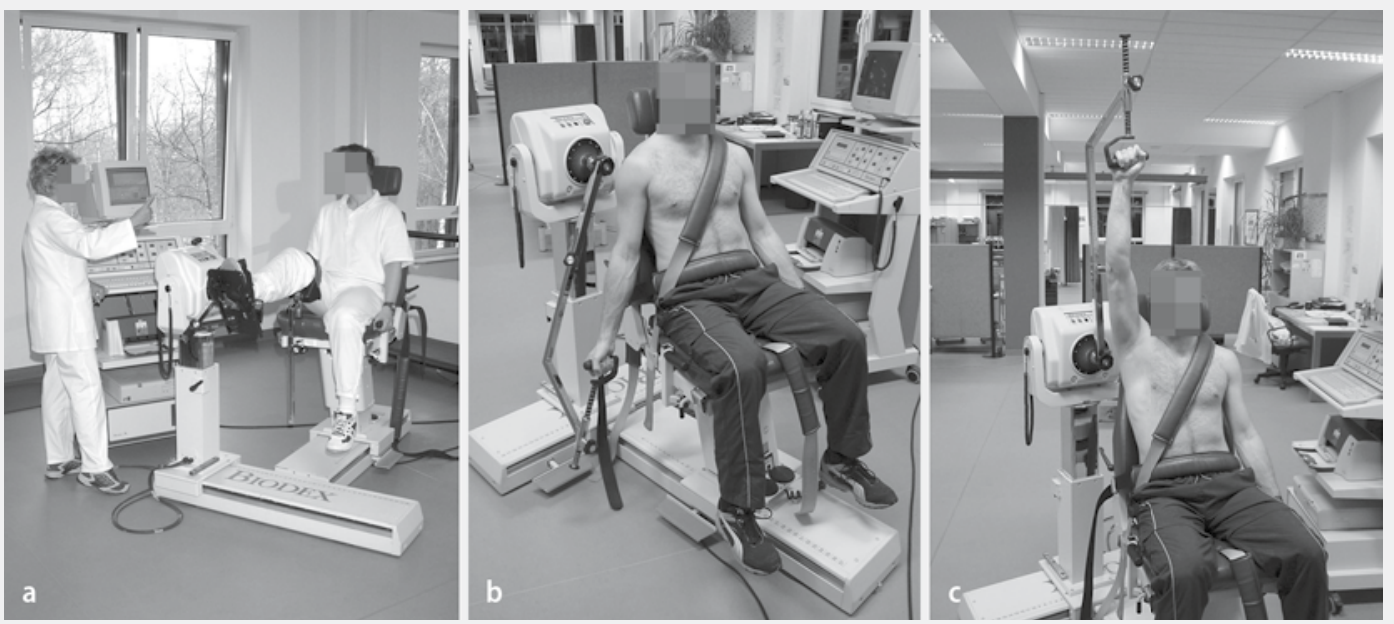

Abb. $3<$ Isokinetik

a Knie-, b,c Schultergelenk 


\begin{tabular}{|ll|}
\hline $\begin{array}{l}\text { Tab. } 2 \\
\text { cycle }\end{array}$ & Einsatzgebiete des „diagnostic \\
\hline $\begin{array}{l}\text { Mobilisati- } \\
\text { onstraining }\end{array}$ & $\begin{array}{l}\text { Isokinetischer Trainingsmodus } \\
\text { ohne Belastungs- und Be- } \\
\text { schleunigungsspitzen }\end{array}$ \\
\hline $\begin{array}{l}\text { Intervall- } \\
\text { training }\end{array}$ & $\begin{array}{l}\text { Aufbautraining mit wechseln- } \\
\text { den Belastungsintensitäten } \\
\text { mit Erholungsphasen }\end{array}$ \\
\hline $\begin{array}{l}\text { Ausdauer- } \\
\text { training }\end{array}$ & $\begin{array}{l}\text { Trainingsprogramme unter } \\
\text { Herzfrequenzkontrolle }\end{array}$ \\
\hline $\begin{array}{l}\text { Leistungs- } \\
\text { training }\end{array}$ & $\begin{array}{l}\text { Leistungssteigerung in der } \\
\text { effektiven Trainingszone unter } \\
\text { Herzfrequenzsteuerung (bis } \\
130 \mathrm{~W})\end{array}$ \\
\hline
\end{tabular}

haben die CPM-Arm- (• Abb. 1) und Bein-Motorschienen (• Abb. 2), die ihre Anwendungspriorität in der unmittelbaren postoperativen Verordnung finden, eine neue Bewertung gefunden, seitdem sie den Patienten auch leihweise für die ambulante Weiterbehandlung verordnet werden können.

Indikationen und Ziele der CPMSchienen-Anwendungen sind:

- Prävention von Verklebungen nach Gelenk- sowie gelenknahen Operationen oder -Verletzungen,

- Verbesserung der lokalen Knorpelnutrition nach arthroskopischen/offenen Interventionen am Gelenkknorpel,

- Stimulation der chondrozytären Matrixsynthese,

- Expression von lysosomalen Enzymen sowie purulentem Exsudat.

Mit der freien Verfügbarkeit der CPMArm- oder Beinmotorschienen sind die Patienten/-innen z. B. nach Hüft-, Knie-, Schulter- oder Ellenbogengelenkoperationen in der Lage, eigenständig in der Regel 2- bis 3-mal täglich über eine Anwendungsdauer von 20-30 min Mobilisierungstherapien selbstbestimmt ab dem ersten postoperativen Tag durchzuführen (• Tab. 1).

\section{Erweiterte Ambulante Physiotherapien (EAP)}

Indikationen für die Verordnungen von EAP sind:

- Frühzeitig erkennbarer Stillstand (<4 Wochen) eines anfänglichen Funktionsgewinnes unter Standardtherapien (KG, PT),

Trauma Berufskrankh 2010 - 12 [Suppl 1]:29-37 DOI 10.1007/s10039-009-1508-z

c) Springer Medizin Verlag 2009

\section{B. Herbst · H.-R. Kortmann \\ Postoperative ambulante Verordnungen}

\section{Zusammenfassung}

Die aktuelle Handlungsanleitung der Deutschen Gesetzlichen Unfallversicherung (DGUV) legt die Art der physikalisch-therapeutischen Maßnahmen im ambulanten berufsgenossenschaftlichen Heilverfahren standardisiert fest; differenziert wird zwischen Verordnungen über Krankengymnastik, Physikalische Therapien [inklusive CPM (",continous passive motion")] und EAP (Erweiterte Ambulante Physiotherapie) mit MTT (Medizinische Trainingstherapie); inklusive Schulter- und Kniegelenkisokinetik , diagnostic cycle", Rückengeräten, Laufband.
Das Training am BTE (Baltimore Technologies Equipment) wird in der Ergotherapie der BG-Unfallklinik Duisburg in der berufsorientierten Rehabilitation eingesetzt. Verordnungen über Ergotherapie sollten zukünftig von der DGUV über ein Standardformblatt ermöglicht werden.

\section{Schlüsselwörter}

Krankengymnastik · Physikalische Therapien . Erweiterte Ambulante Physiotherapie (EAP). Medizinische Trainingstherapie (MTT) .

Baltimore Technologies Equipment (BTE)

\section{Postoperative outpatient prescriptions}

\section{Abstract}

The current procedural guidelines of the German Social Accident Insurance (DGUV) determine the way in which physical therapeutic measures are prescribed for the treatment of outpatients following accidents which fall upon the insurance of the employers' association; a distinction is made between prescriptions for physiotherapy, physical therapy (including CPM, continuous passive motion), and extended outpatient physiotherapy with MTT (medical training therapy, including shoulder-/knee-joint isokinetics, diagnostic cycle, back trainers and treadmills). Train- ing using BTE (Baltimore Technologies Equipment) is used for occupational therapy and rehabilitation at the statutory accident insurance trauma clinic in Duisburg. Occupational therapy prescriptions should be made possible by the German Social Accident Insurance (DGUV) using a standard form.

\section{Keywords}

Physiotherapy - Physical therapies .

Extended outpatient physiotherapy · Medical training therapy (MTT) .

Baltimore Technologies Equipment (BTE) 
Postoperative ambulate Verordnungen
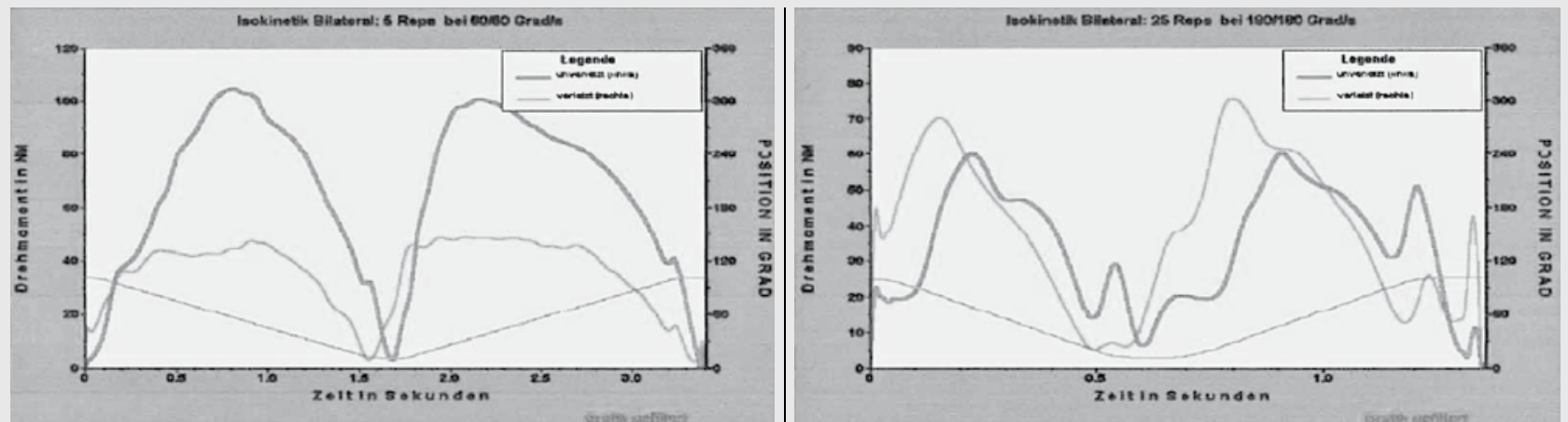

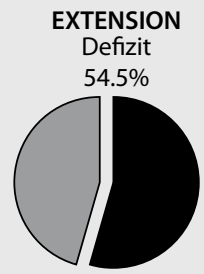

$60 \mathrm{Grad} / \mathrm{s}$

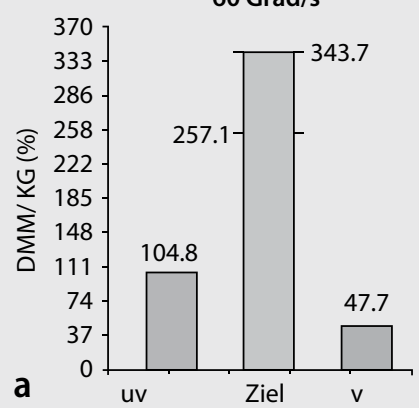

leokinetik Bilatoral: 6 Ropa bei E0ro Gradto

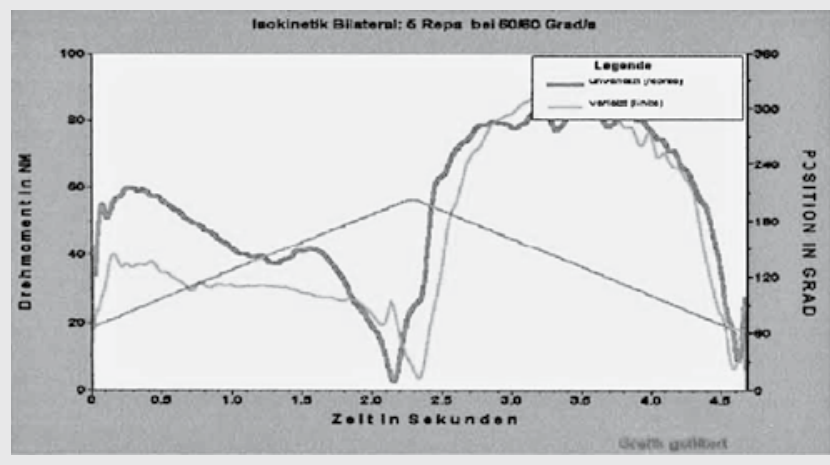

ABDUKTION

Defizit

$33.0 \%$
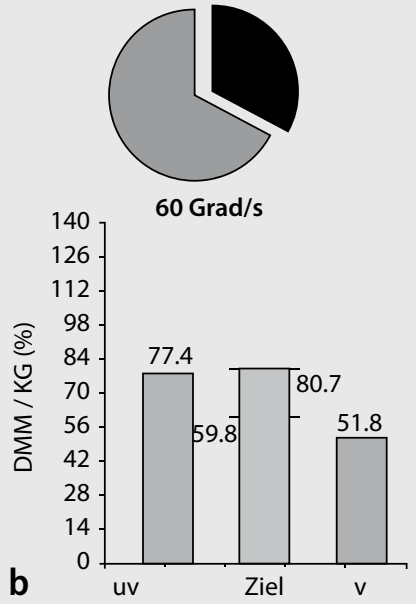

ADDUKTION

stärker
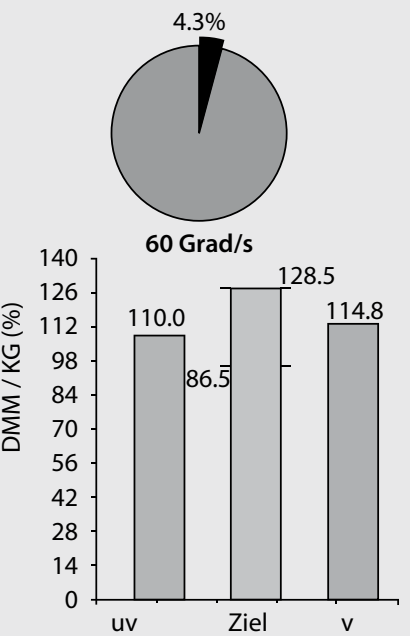
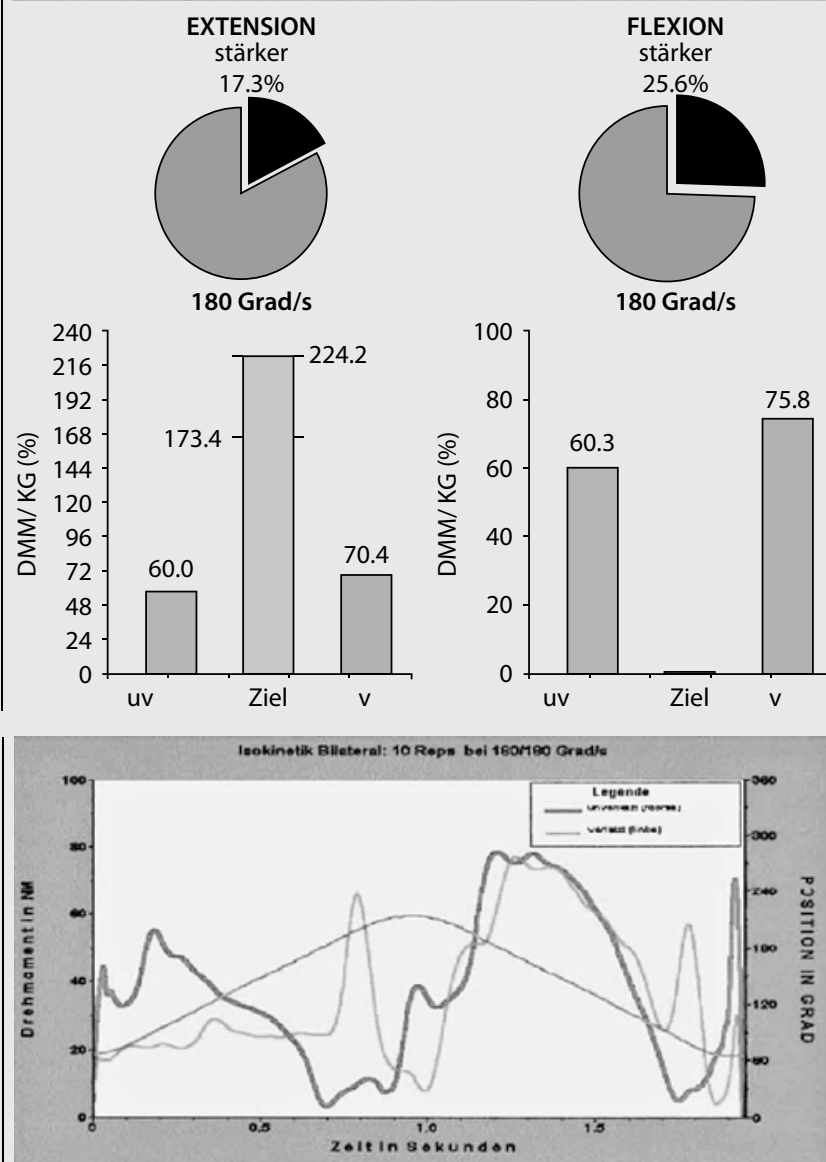

ABDUKTION

stärker
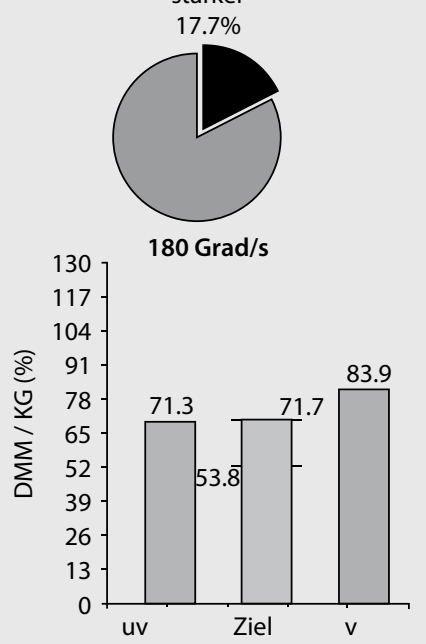
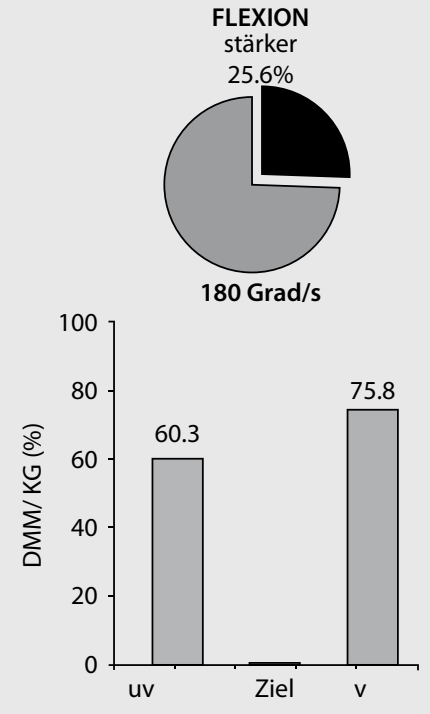

ADDUKTION

Defizit
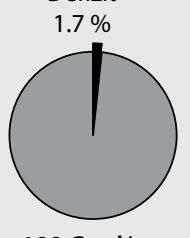

$180 \mathrm{Grad} / \mathrm{s}$

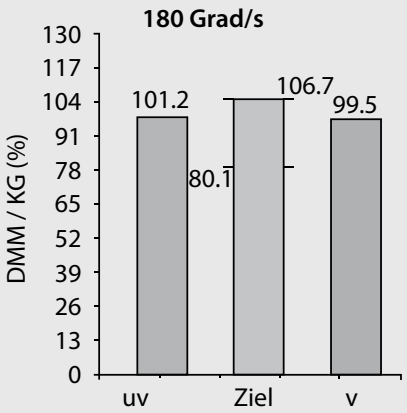




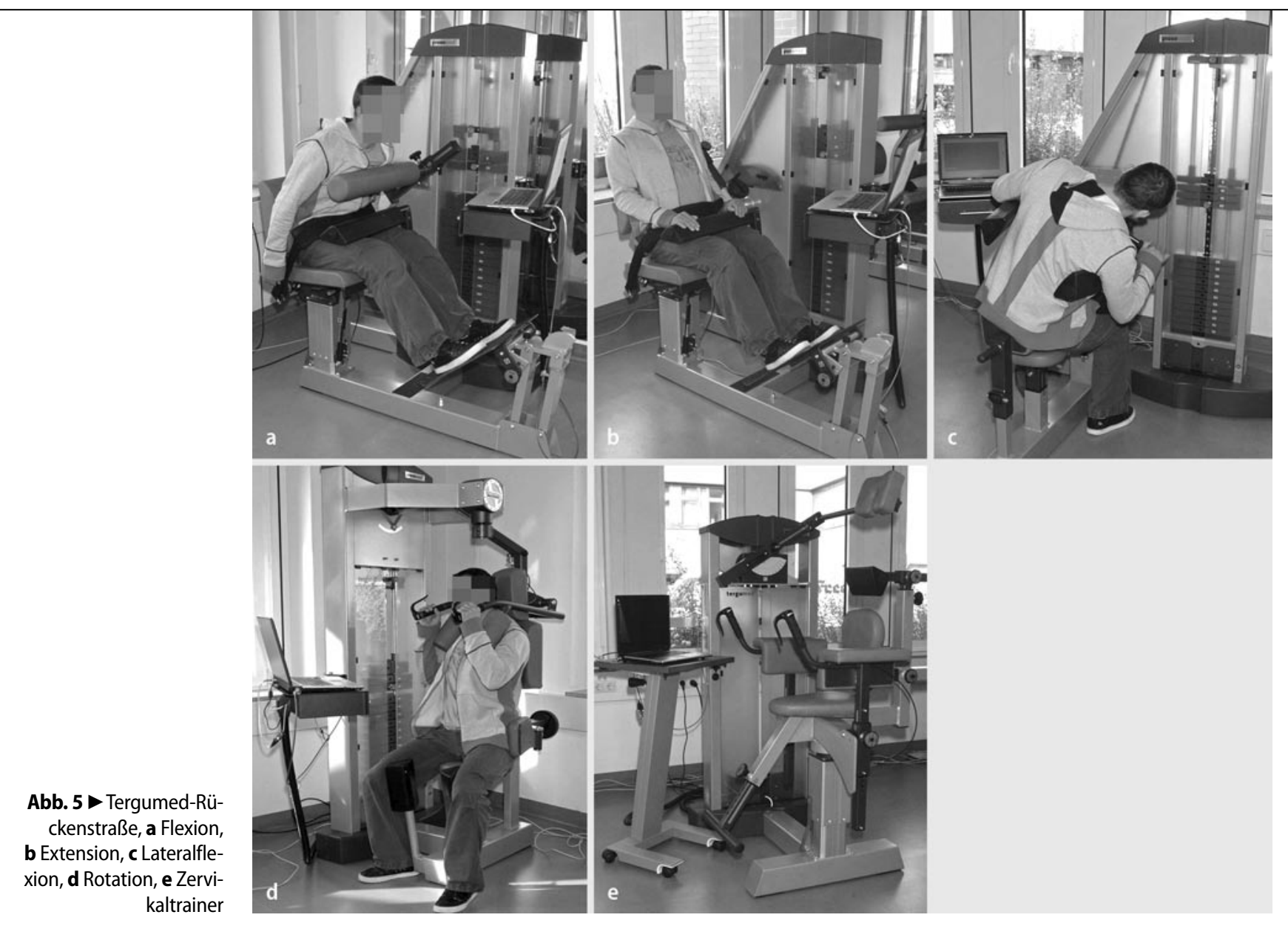

- Bewegungseinschränkungen nach Gelenk(teil)steifen,

- komplexe Gelenkverletzungen mit verzögerter Mobilisierbarkeit,

- objektiv nachweisbare Muskelschwächen oder Muskelfunktionsstörungen nach Verletzungen und/oder Operationen,

- koordinative Leistungsschwächen.

Mit dem Formblatt F2410 der DGUV sind die Verordnungen zur Durchführung Erweiterter Ambulanter Physiotherapien in einer lizensierten EAP-Einrichtung standardisiert festgelegt.

Die EAP-Verordnungen beinhalten KG, PT und MTT, wobei Letztere auch innerhalb der EAP isoliert verordnet werden kann. Das Indikationsspektrum für die MTT umgreift neben der postoperativen ambulanten Rehabilitation auch Prävention und Fitness bis hin zum pro-

Abb. 4 < Isokinetiktestauswertungen, a Knie-, b Schultergelenk fessionellen Leistungssport. Die verordneten Therapien setzen ein, wenn die Kostenübernahmeerklärung des Unfallversicherungsträgers vorliegt. Eine Einzelverordnung umfasst Therapien über 2 Wochen; zur Fortführung ist eine Neuverordnung des D- oder H-Arztes notwendig. Therapieziel sind die Steigerung von $\mathrm{Be}$ weglichkeit, Kraft, Koordination und Ausdauer. Die EAP-Behandlungssequenzen umfassen 4-6 Behandlungstage/Woche. Ärztliche Kontrolluntersuchungen sind längstens 14 Tage nach Behandlungsbeginn vorgesehen.

\section{Knie-/Schultergelenkisokinetik}

Isokinetik bedeutet: gleichförmige Bewegung, bei sich ständig direkt oder indirekt anpassendem Widerstand.

Hislop u. Perrine [2] entwickelten 1967 das erste isokinetische Testgerät. Sie verfolgten das Ziel, ein System unter einer im Voraus festgelegten Bewegungsgeschwindigkeit und gegen einen Widerstand, der sich mittels elektronisch gesteuerter Me- chanik und Hydraulik während der gesamten Bewegung aufbaut, zu entwickeln (- Abb. 3).

Zielsetzungen der Isokinetik sind:

- Erfassung funktioneller Störungen der Gelenkmechanik und muskulärer Defizite,

- Objektivierung schmerzbedingter Funktionseinschränkungen,

- Beurteilung und Steuerung von Therapie- und Trainingsbelastungen,

- Erfassung von Richt- und Orientierungswerten.

\section{Testauswertungen}

Das maximal erreichte Drehmoment, die Drehmomentkurve über den gesamten Bewegungsablauf, die durchschnittliche sowie Gesamtarbeit und Leistung, die explosive Komponente und Ausdauer können quantitativ bestimmt und die Quotienten Agonist/Antagonist, gesundes/verletztes Gelenk, Patientenleistung/Durchschnittskollektiv analysiert werden. Primärtests (rechts/links), Vergleichstests und Vorbefunde (re-test) sowie Längs- 


\section{Postoperative ambulate Verordnungen}

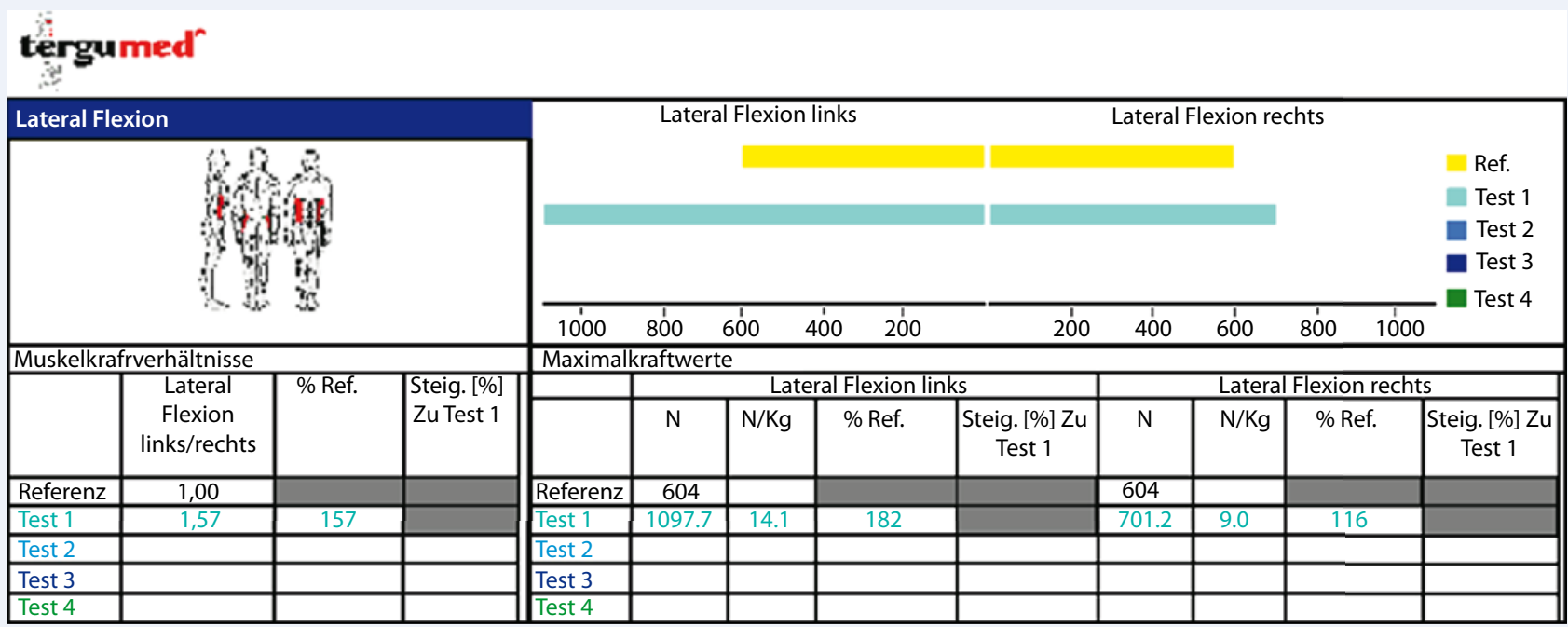

a

Kraftanalyse in graphischer Drastellung

\begin{tabular}{|c|c|c|c|c|c|c|c|c|c|c|}
\hline & Extension & Flexion & \multicolumn{2}{|c|}{ Rotation } & \multicolumn{2}{|c|}{ Lateralflexion } & \multicolumn{2}{|c|}{ HWS Wxtension : Flexion } & \multicolumn{2}{|c|}{ HWS Lateralflexion } \\
\hline & & & links & rechts & links & rechts & links & rechts & links & rechts \\
\hline $\begin{array}{l}\text { über Ref.- } \\
\text { bereich }\end{array}$ & & 0 & & & & 0 & & & & \\
\hline Referenz & & & & & & & & & & \\
\hline $\begin{array}{l}\text { Unter Ref.- } \\
\text { bereich }\end{array}$ & 0 & & 0 & 0 & & & & & & \\
\hline
\end{tabular}

\section{Bewertung:}

Die Grafik zeigt das indiciduell in der Messung erreichte Kraftprofil im Vergleich zu Referenzdaten auf. Form und Verlauf der Linien bewerten das erreichte Kraftprofil im Vergleich zur Referenz (Kästchenline im Referenzbereich).

Kraftverhältnis in \%

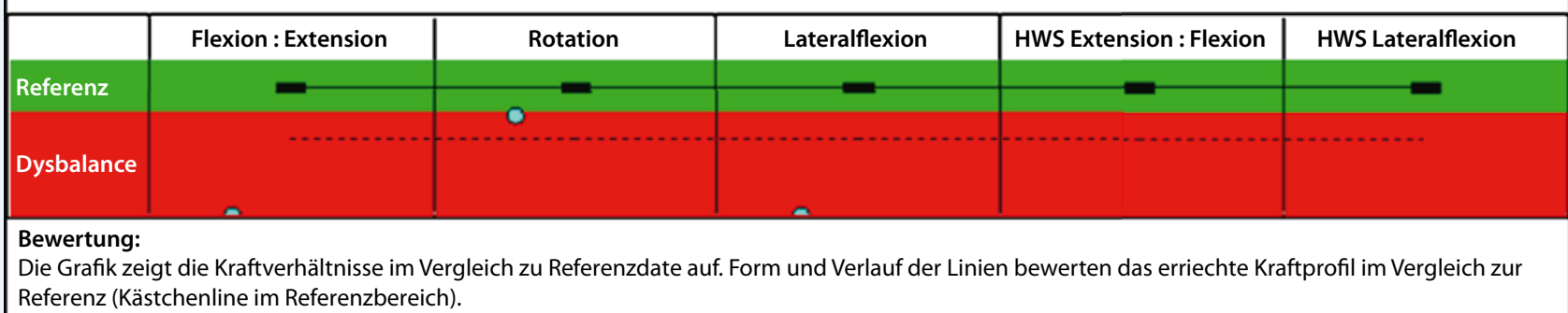

b

Abb. $6 \Delta$ Tergumend-Softwaremodul-Auswertung, a Analyse der Kraftwerte, b Analyse der Kraftwerte und Dysbalancen

schnittbeurteilungen bei fortgesetztem Therapieeinsatz sind ebenfalls möglich (- Abb. 4). Bei der visuellen Kurvenbeurteilung wird auf ein annähernd identisches Kurvenbild geachtet; ein harmonischer Kurvenverlauf oder Dellen, Einbrüche oder Zackenbildungen werden in Art und Lokalisation beschrieben.

Folgende Fragestellungen sind bei der Analyse zu beantworten:

Warum ist die Drehmomentkurve erniedrigt? Ursachen können sein:

- muskuläre Atrophien,

- neurogene Störungen
- Erguss und/ oder Kapselschwellung,

- metabolische Störungen (gestörter Blut-/Lymphfluss, Entzündungsstoffwechsel).

Warum gibt es Einbrüche in der Drehmomentkurve? Reproduzierbare Ursachen sind:

- Arthrose,

- Schmerz,

- Störungen der Gelenkmechanik und

- gerätetechnische Probleme.

Nicht reproduzierbare Ursachen sind:
- spontane Störungen der Gelenkmechanik sowie

- persönliche Faktoren.

Warum sind Veränderungen der Drehmomentkurven über die Zeit erkennbar? Abfallende Drehmomentkurven zeigen:

- muskuläre Ermüdung oder Ausbelastung.

Stagnierende Drehmomentkurven sind Hinweise auf:

- inhibitorische Prozesse, 
- Motivationsprobleme,

- Angst,

- mangelnde Gerätegewöhnung,

- mangelnde Instruktion,

- Schmerz und/oder

- intraartikuläres Druckgefühl.

\section{"diagnostic cycle"}

Er stellt eine weitere Möglichkeit des computergestützten Gerätetrainings dar und kommt nach einem Eingangstest zur Ermittlung der aktuellen Leistungsfähigkeit (Aussagekraft hinsichtlich Belastbarkeit anhand Herzfrequenz und Wattzahl) laut der in 0 Tab. 2 dargestellten Einsatzgebiete zur Anwendung.

\section{Tergumed-Rückenstraße}

Die verschiedenen Test- und Trainingsstationen in der Rückenstraße sind durch ihre unterschiedlichen Gerätekonstruktionen auf die einzelnen Funktionen der menschlichen Wirbelsäule abgestimmt. Indikationen für ihren Einsatz sind:

- Wirbelsäulenverletzungen,

- schmerzhafte Wirbelsäulensymptomatiken,

- Bandscheibenprotrusionen und -vorfälle,

- chronische Bandscheibenleiden,

- degenerative Wirbelsäulenveränderungen,

- Spondylosen,

- Spondylolisthesen,

- Wirbelsäulenverkrümmungen (Skoliosen, Kyphosen).

Das Tergumed Flexion ( $\bullet$ Abb. 5a) wird zum Training der Bauchmuskulatur (Mm. rectus abdominis, obliquus externus abdominis et internus abdominis), das Tergumed Extension ( $\bullet$ Abb. 5b) zum Training der Rückenstreckmuskulatur (Mm. erectores, longissimi und interspinales), das Tergumed Lateralflexion ( $\bullet$ Abb. 5c) zum Training der lateralen Rumpfmuskulatur (Mm. quadratus lumborum, obliquus externus abdominis et internus abdominis und autochthone Muskulatur) und das Tergumed Rotation (• Abb. 5d) zum Training der schrägen und tief liegenden Bauchmuskulatur (Mm. obliquus externus abdominis et internus abdomi-
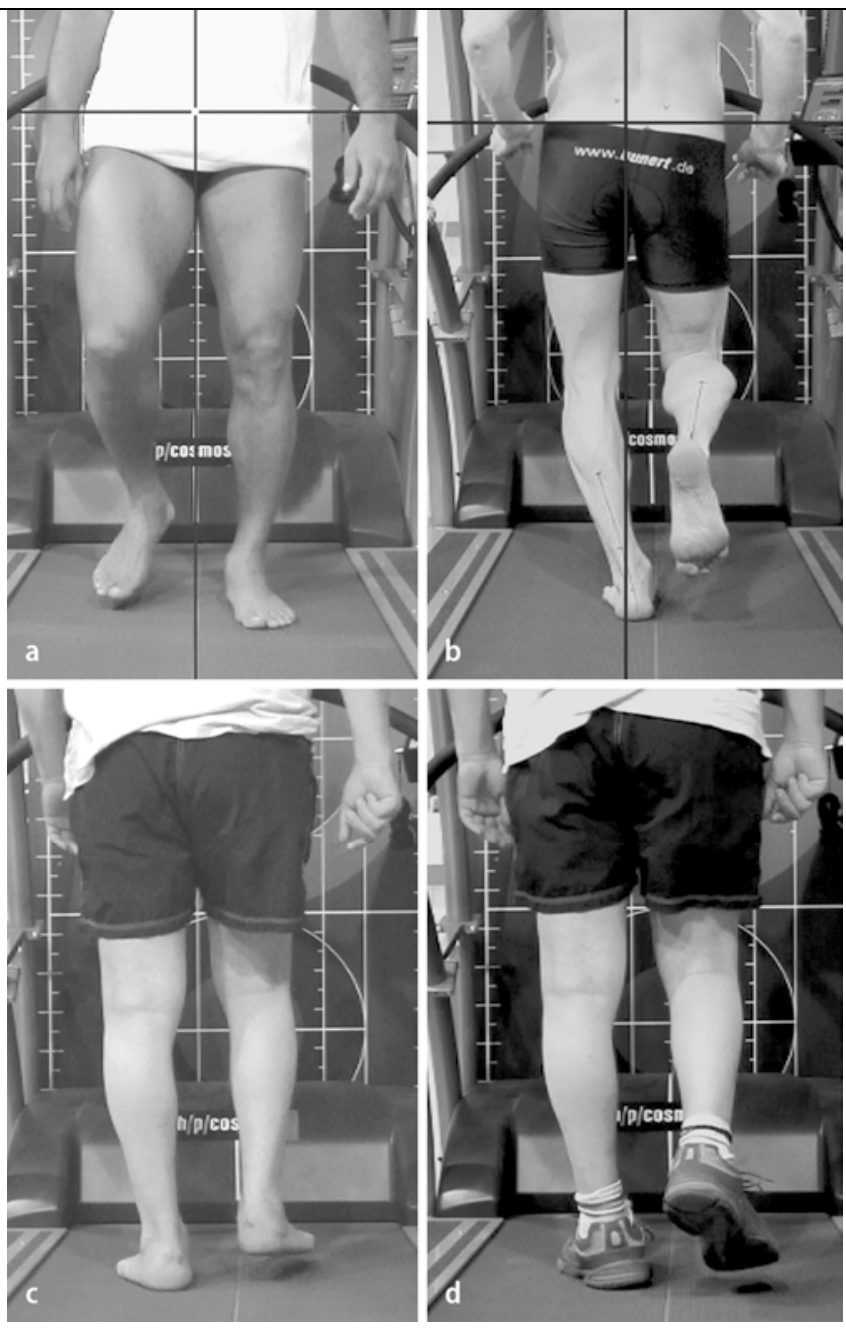

Abb. 7 Gangbildanalyse, a achsausgerichtetes Barfußgangbild, $\mathbf{b}$ varische Beinachsabweichung, Tiefertreten der Beckenseite, c eingesunkenes Fußgewölbe, Pronations-/Abduktionsstellung, $\mathbf{d}$ durch seitlich ausgetretenes, forminstabiles Turnschuhwerk verstärkte Supinationsfehlstellung

nis, rotatores und autochthone Muskulatur) genutzt.

Der Tergumed Zervikaltrainer (- Abb. 5e) wird zum Training folgender Muskeln eingesetzt:

- Extension: Mm. splenii capitis et cervicis, rectus capitis posterior, minor et major obliquus capitis superior et inferior,

- Flexion: Mm. sternocleidomastoidei longus colli et capitis und rectus capitis anterior,

- Lateralflexion: Mm. splenii capitis et cervicis, rectus capitis lateralis, $\mathrm{Mm}$. sternocleidomastoidei, scalenus anterior medius et posterius, longus colli et capitis.

Die Tergumed-Softwaremodule liefern grafische Standarddarstellungen der Testauswertungen für Kraftwerte und Dysbalancen sowie Verlaufsberichte zur Erfolgskontrolle. Die Ergebnisse der Muskelkraftund Bewegungsbereichsmessung werden für jedes Gerät numerisch und auch grafisch dargestellt (• Abb. 6).

Die Darstellung der Gesamtbewertung und des neuromuskulären Profils ermöglicht einen präzisen Überblick über das komplette Kraftprofil der wirbelsäulenstabilisierenden Muskulatur im Vergleich zu Referenzdaten. Zusätzlich wird das Kraftverhältnis der antagonistisch arbeitenden Muskulatur im Vergleich zu gesunden Probanden aufgezeigt. Die farbliche Abstufung macht bestehende Dysbalancen deutlicher (• Abb. 6).

\section{Videogestützte Laufbandanalysen}

Die Physiologie des menschlichen Gangbildes ist in 3 Phasen zu unterteilen:

1. Landephase, die mit $0,05 \mathrm{~S} 20 \%$ der Belastungsphase des Fußes beim Gehen ausmacht; dabei wirkt bei der Landung der Ferse das 3 1/2- bis 5Fache des Körpergewichtes auf Fuß und Körper. 


\section{Postoperative ambulate Verordnungen}
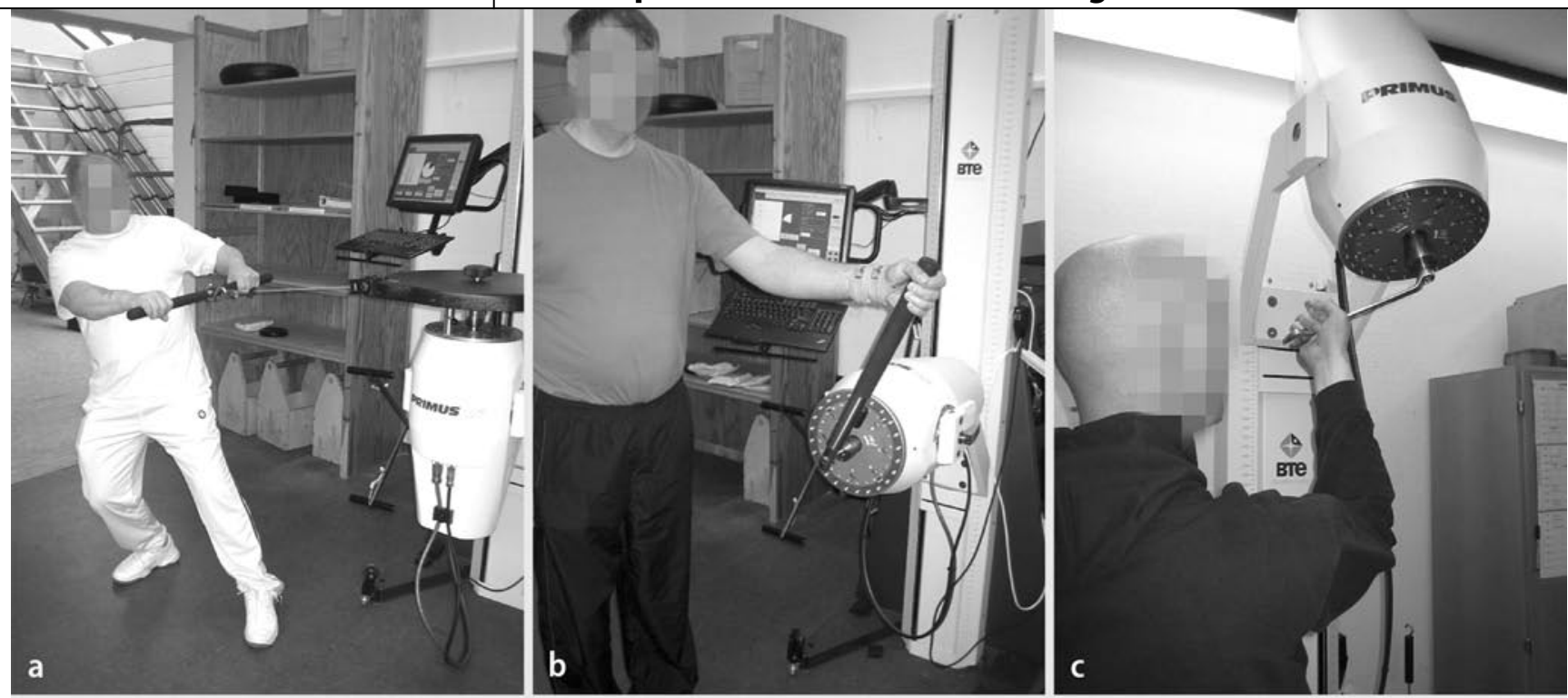

BTE Technologies Evaluation Results

Report

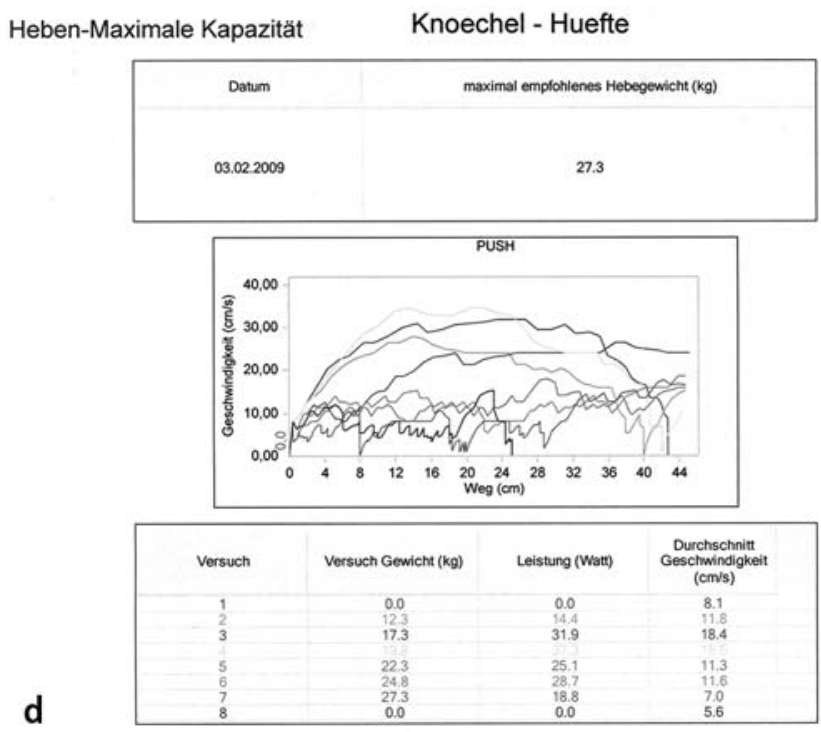

Abb. 8 \ BTE-Gerät, Simulation von a Zieh-, b Schiebevorgängen, c Maschinenhebelbedienung, d Auswertungsbeispiele

2. Stützphase, die mit 0,088 s $30 \%$ der Belastungsphase ausmacht: Der ganze Fuß berührt den Boden, die Pronationsbewegung vergrößert die Standfläche des Fußes und sichert diesen gegen Umknicken nach außen. Die Landephase (Dämpfung) ist insgesamt verzögert und bringt die Beinachse in ihre Arbeitsposition.

3. Abdruckphase, die mit 0,15 s $50 \%$ der Belastungsphase ausmacht: Bis zum 5-Fachen des Körpergewichtes wirkt auf die Mittelfußköpfchen, bei Abdruck über die Großzehe mit Druckmaximum bei Mittelfußköpfchen 2-5.
Zielsetzungen für die videogestützte Gangbildanalyse (• Abb. 7) sind:

- Erstellen individueller Laufanalysen (Physiologie der Körperachsen),

- Analyse gangspezifischer Funktionen (Barfuß-/Sohlengang, Geh-/Laufschritt, ebenerdig/Anstieg - Gefälle),

- Informationsdatenbank für die häufigsten gang- und laufspezifischen Beschwerden (Pes, Genu, Coxa, Vara/ Valga). 
- Wirbelsäulenverletzungen und

- Gliedmaßenamputationen.

Methodisch eignet es sich für:

- isometrische Krafttests im bilateralen und im Agonisten-Antagonisten-Vergleich,

- isotonische Leistungstests, wie Drehmoment/Geschwindigkeit,

- Ausdauertests,

- isometrische Liniengrafiktests,

- isokinetische Tests sowie

- Tests der maximalen Anstrengungsbereitschaft.

Ziele sind:

- aktive Therapien mit funktioneller Ausrichtung,

- $\operatorname{ADL}($,activities of daily living“)-Training,

- arbeitsplatzspezifische Rehabilitation,

- Verbesserung der sportspezifischen Leistungsfähigkeit.

Das BTE-Gerät wird in der BG-Unfallklinik Duisburg seit vielen Jahren mit Erfolg im Rahmen der EAP/MTT-Verordnungen von den Ergotherapeuten eingesetzt und hat sich in der letzten Stufe der berufsorientierten Rehabilitation bewährt. Für die Zukunft wäre deshalb zu wünschen, dass die DGUV mit Festlegung eines Verordnungsformblattes für die Ergotherapie auch dieses Trainingsgerät als medizinischen Standard anerkennt.

\section{Korrespondenzadresse}

\section{Dr. B. Herbst}

BG-Unfallklinik Duisburg GmbH, Großenbaumer Allee 250, 47249 Duisburg

barbara.herbst@bgu-duisburg.de

Interessenkonflikt. Die korrespondierende Autorin gibt an, dass kein Interessenskonflikt besteht.

\section{Literatur}

1. DGUV (2008) DGUV Handlungsanleitung vom 01.01.2008 des Landesverbandes West Düsseldorf. Landesverband West, Düsseldorf

2. Hislop HJ, Perrine JJ (1967) The isokinetic concept of exercise. Phys Ther 47(2):114-117 